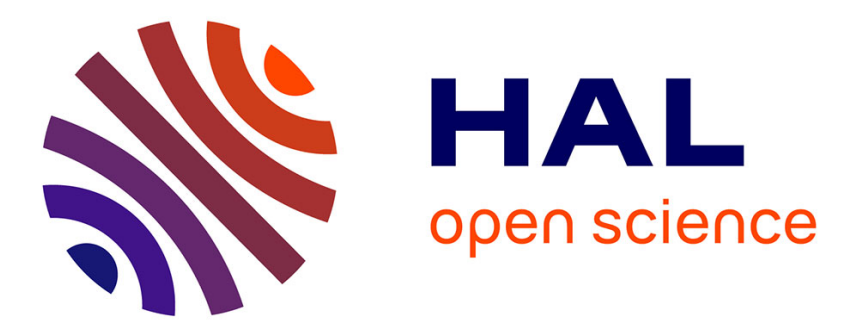

\title{
A method to avoid the unmeasurable premise variables in observer design for discrete time TS systems
}

Dalil Ichalal, Benoît Marx, Didier Maquin, José Ragot

\section{To cite this version:}

Dalil Ichalal, Benoît Marx, Didier Maquin, José Ragot. A method to avoid the unmeasurable premise variables in observer design for discrete time TS systems. International Conference on Fuzzy Systems, FUZZ-IEEE 2016, Jul 2016, Vancouver, Canada. pp.2343-2348, 10.1109/FUZZ-IEEE.2016.7737986 . hal-01288326

\section{HAL Id: hal-01288326 \\ https://hal.science/hal-01288326}

Submitted on 25 Nov 2021

HAL is a multi-disciplinary open access archive for the deposit and dissemination of scientific research documents, whether they are published or not. The documents may come from teaching and research institutions in France or abroad, or from public or private research centers.
L'archive ouverte pluridisciplinaire HAL, est destinée au dépôt et à la diffusion de documents scientifiques de niveau recherche, publiés ou non, émanant des établissements d'enseignement et de recherche français ou étrangers, des laboratoires publics ou privés. 


\title{
A method to avoid the unmeasurable premise variables in observer design for discrete time TS systems
}

\author{
D. Ichalal, B. Marx, D. Maquin, J. Ragot
}

\begin{abstract}
This paper addresses a new method to overcome the unmeasurable premise variables in TS nonlinear discrete time systems for observer design. It is known that a TS system can be obtained directly from a nonlinear one by using the sector nonlinear transformation in a given compact set of the state space. However, this procedure often leads to TS systems having premise variables depending on one or more unmeasurable states. Clearly, the observer design for such a class of TS systems is more difficult than designing observers for TS systems having measured or known premise variables. The objective of this paper is to use the immersion approach before using the sector nonlinear transformation in order to obtain a TS system with measured premise variables. In addition the proposed immersion technique leads to the use of an augmented state vector which first components are directly the state variables of the original system avoiding the computation of an inverse transformation. Example results are provided in order to illustrate the proposed approach.
\end{abstract}

\section{INTRODUCTION}

Using Takagi-Sugeno system theory in control and observation is one of the most explored approaches to handle nonlinear systems in the last forty years from the seminal work of [14]. The interest of the TS structure is in its simple formulation of the nonlinear system in a polytopic form [15]. Indeed, such a structure allows the extension some tools and theories from linear system domain to the nonlinear one such as LMIs, Lyapunov stability analysis, control and observation. This results to an active domain of research for nonlinear systems in both theoretical and application point of views. The TS systems can be obtained by different ways, namely, the identification techniques from a set of input output measurements and fixed model structure [5], the linearization around several operating points judiciously chosen and interpolated by adequate nonlinear functions satisfying the convex sum property [13], and the transformation from a nonlinear system by using the sector nonlinear transformation [15]. The present work focuses on the last method of obtaining TS systems.

Using the sector nonlinear transformation, two types of TS systems can be obtained: the first one presents TS systems having measurable premise variables (which depend on only measured variables). The second type consists in TS systems having premise variables depending, partly or completely, on unmeasured states of the system. In the context of state

D. Ichalal is with IBISC-Lab, Evry-Val d'Essonne University, 40, rue de Pelvoux, 91020, Courcouronnes, France, dalil.ichalal@ibisc.univ-evry.fr

B. Marx, D. Maquin and J. Ragot are with Université de Lorraine, Centre de Recherche en Automatique de Nancy, CNRS, CRAN, UMR 7039, 2 avenue de la Forêt de Haye, 54516 Vandoeuvre-lès-Nancy, France. observer design, the first class of TS systems is largely explored and several results are provided, which is not the case for the second class of TS systems. Nevertheless, one can cite some results for such a class of TS systems, for example, the first work dealing with this problem has been reported in [1] which extends the well known ThauLuenberger observer [16] by using Lipschitz conditions on the perturbation-like term due to the unmeasured premise variables. This idea has been exploited in [12] for cascaded systems and in [10] by assuming the Lipschitz conditions of the weighting functions rather than the global Lipschitz condition of the perturbation-like term. However, taking into account the Lipschitz constants limits the applicability of the approaches because the LMI conditions depend on the Lipschitz constants and introduce a severe conservatism in the existence of a solution to these LMIs. In order to relax the conservatism of the Lipschitz-based approach, in [8], [6], a new approach has been proposed by using the vector differential mean value theorem. This approach reduces significantly the conservatism related to the Lipschitz constant but it may introduce a huge number of sub-models which increases the computational complexity. More recently, other approaches [11], [17] are provided to overcome the problems of the cited techniques, it consists in leaving the asymptotic or exponential convergence property of the state estimation error and guaranteeing only bounded state estimation error by using $\mathscr{L}_{2}$ and Input-to-State Stability concepts (ISS). The main advantage of such results is the obtention of LMIs free from the Lipschitz constants and without adding additional sub-models. The notion of bounded state estimation error can also be found in [9] where the state estimation error is transformed into a TS form with polytopic uncertainties due to the unmeasurable premise variables.

In the context of discrete time TS systems with unmeasurable premise variables, observation problem is not largely studied which motivates the result of the present paper. The main idea of this paper is to obtain a way to transform the TS system with unmeasurable premise variables into an equivalent TS system with measurable premise variables in order to apply existing results.

From the above analysis, it can be seen clearly that the observer design for TS systems with unmeasurable premise variables is more complex and far from to be solved compared to the case of TS systems with unmeasurable premise variables. It is then natural to ask the following question: Before using the nonlinear sector transformation, is it possible to transform, equivalently, the system in order to express it into an quasi-LPV form where the parameters depend only 
on measured variables? Of course, if such a transformation exists, the use of the nonlinear sector transformation leads to a TS system with measurable premise variables, and then, the techniques developed for such systems can be applied. This paper presents a first solution for this problem by using dynamic state extension inspired from the immersion techniques used in the nonlinear framework (see [2] and references therein). Notice that, the proposed transformation does not need the computation of the inverse transformation to get the original state because this last is contained in the new state vector.

\section{MAIN RESULT}

Let us consider the nonlinear system in the form

$$
\left\{\begin{array}{l}
x_{k+1}=f\left(x_{k}\right)+g\left(x_{k}\right) u_{k} \\
y_{k}=C x_{k}
\end{array}\right.
$$

where $x_{k} \in \mathbb{R}^{n}, u_{k} \in \mathbb{R}^{n_{u}}$ and $y_{k} \in \mathbb{R}^{n_{y}}$ represents, respectively, the state, the input and the output vectors of the system. $f$ and $g$ are vector functions depending on the state vector $x_{k}$ and $f(0)=0 . C$ is a known matrix with appropriate dimensions.

Classically, the sector nonlinear transformation is applied to obtain an exact TS system in a compact set $\Omega$ of the state space as follows [15]

$$
\left\{\begin{array}{l}
x_{k+1}=\sum_{i=1}^{r} h_{i}\left(\xi_{k}\right)\left(A_{i} x_{k}+B_{i} u_{k}\right) \\
y_{k}=C x_{k}
\end{array}\right.
$$

where $\xi_{k}$ is the vector of premise variables and the matrices $A_{i}$ and $B_{i}$ are with appropriate dimensions and correspond to the $i^{t h}$ sub-model of the TS system. $r$ is the number of submodels and the functions $h_{i}\left(\xi_{k}\right)$ are the weighting functions depending on the premise variables $\xi_{k}$ and satisfying the convex sum property

$$
\left\{\begin{array}{l}
\sum_{i=1}^{r} h_{i}\left(\xi_{k}\right)=1 \\
0 \leq h_{i}\left(\xi_{k}\right) \leq 1, \forall k, i=1, \ldots, r
\end{array}\right.
$$

This transformation often leads to TS system where the premise variables $\xi_{k}$ depend partly or completely on unmeasured states. As explained in the introduction, designing an observer when the premise variables are unmeasurable is more difficult. The idea is then to transform the nonlinear system into the form

$$
\left\{\begin{array}{l}
z_{k+1}=A\left(y_{k}, u_{k}\right) z_{k}+B\left(y_{k}, u_{k}\right) u_{k} \\
y_{k}=\mathscr{C} z_{k}
\end{array}\right.
$$

where $z_{k} \in \mathbb{R}^{N}$ is a new state vector of dimension $N \geq n$ where the components of $x_{k}$ are contained in $z_{k}$. Consequently, if $z_{k}$ is estimated the original state $x_{k}$ is obtained directly from $z_{k}$. The system (4) can then be expressed in TS form by using the nonlinear sector transformation. One obtains

$$
\left\{\begin{array}{l}
z_{k+1}=\sum_{i=1}^{r^{\prime}} h_{i}\left(y_{k}, u_{k}\right)\left(\mathscr{A}_{i} z_{k}+\mathscr{B}_{i} u_{k}\right) \\
y_{k}=\mathscr{C} z_{k}
\end{array}\right.
$$

where the matrices $\mathscr{A}_{i}, \mathscr{B}_{i}$ and $\mathscr{C}$ are with appropriate dimensions and the weighting functions depend only on the measured variables $y_{k}$ and $u_{k} . r^{\prime}$ represents the number of the sub-models of the new TS model. In such a case the proportional observer takes the form

$$
\left\{\begin{array}{l}
\hat{z}_{k+1}=\sum_{i=1}^{r^{\prime}} h_{i}\left(y_{k}, u_{k}\right)\left(\mathscr{A}_{i} \hat{z}_{k}+\mathscr{B}_{i} u_{k}+\mathscr{L}_{i}\left(y_{k}-\hat{y}_{k}\right)\right) \\
\hat{y}_{k}=\mathscr{C} \hat{z}_{k}
\end{array}\right.
$$

By defining the state estimation error $e_{k}=z_{k}-\hat{z}_{k}$, its dynamics obeys to the difference equation

$$
e_{k+1}=\sum_{i=1}^{r^{\prime}} h_{i}\left(y_{k}, u_{k}\right)\left(\mathscr{A}_{i}-\mathscr{L}_{i} \mathscr{C}\right) e_{k}
$$

The stability of such error system is largely studied and interesting results are provided based on LMI formalism in order to design the gains $\mathscr{L}_{i}$ [4], [3]. These LMIs are obtained by using different LMI conditions and relaxation mechanisms.

\section{A. System transformation by state extension}

In this section, the approach to transform the system (1) into the form (4) will be given.

1) Step 1: Initialize the first new variables from the state vector $z_{k}^{(i)}=x_{k}^{(i)}, i=1, \ldots, n$.

2) Step 2: For each new variable, compute its value at $k+1$ (exactly the same equations as the original system (1)) and separate all the functions depending only on measured variables $y_{k}$ and $u_{k}$ and define the remaining nonlinear functions as new variables $z_{k}^{l}, l>n$. By computing the values of these variables at $k+1$ it follows

$$
\begin{aligned}
z_{k+1}^{(l)} & =\sum_{i=1}^{l} a_{l, i}\left(y_{k}, u_{k}\right) z_{k}^{(i)} \\
& +\sum_{i=l+1}^{S} a_{l+1, i}\left(y_{k}, u_{k}\right) z_{k}^{(i)}+\varphi_{l}\left(y_{k}, u_{k}\right)
\end{aligned}
$$

where $z_{k}^{(i)}, i=l+1, \ldots, s$ denote other defined new variables. The functions $a_{l, i}, a_{l+1, i}$ and $\varphi_{l}\left(y_{k}, u_{k}\right)$ are scalar and depend only on measured variables. The step 2 is repeated for all the defined variables and the parameter $s$ converges, if a transformation exists, to $N$ the dimension of the new state vector $z_{k}$.

3) Step 3: The algorithm stops when the time derivative of the $l^{\text {th }}$ new state is free from nonlinear functions depending on the unknown states.

By using this procedure, the system described in equation (4) is obtained and by using the sector nonlinear transformation in a compact set, one obtains the TS system (5) having only measurable premise variables. Then the design of the observer follows the classical approach. 
Example: Let us consider the nonlinear discrete time system derived from the Duffing map

$$
\left\{\begin{array}{l}
x_{k+1}^{(1)}=x_{k}^{(2)} \\
x_{k+1}^{(2)}=-2\left(x_{k}^{(1)}\right)^{3}+2 x_{k}^{(1)}+0.3 x_{k}^{(1)} x_{k}^{(2)} \\
y_{k}=x_{k}^{(2)}
\end{array}\right.
$$

It is clear that using directly the sector nonlinear transformation leads, inevitably, to a TS system with unmeasurable premise variables. As an example, one has the following quasi-LPV system

$$
x_{k+1}=\left[\begin{array}{cc}
0 & 1 \\
0.3 x_{k}^{(2)}+2-2\left(x_{k}^{(1)}\right)^{2} & 0
\end{array}\right] x_{k}
$$

By considering the premise variable $\xi_{k}=0.3 x_{k}^{(2)}+2-$ $2\left(x_{k}^{(1)}\right)^{2}$, a TS model can be obtained. However, the premise variable depend on the unmeasured state $x_{k}^{(1)}$ which complicates the design of a TS observer. As the new state is free from nonlinear function of the unmeasured states, the algorithm stops.

Now, by using the proposed approach, a TS model with premise variables depending only on measured variables is obtained as follows:

1) Step 1: Initialize the new variables $z_{k}^{(1)}=x_{k}^{(1)}$ and $z_{k}^{(2)}=x_{k}^{(2)}$. One obtains the system

$$
\left\{\begin{array}{l}
z_{k+1}^{(1)}=z_{k}^{(2)} \\
z_{k+1}^{(2)}=-2\left(z_{k}^{(1)}\right)^{3}+2 z_{k}^{(1)}+0.3 z_{k}^{(1)} z_{k}^{(2)}
\end{array}\right.
$$

which can be expressed as follows

$$
\left\{\begin{array}{l}
z_{k+1}^{(1)}=z_{k}^{(2)} \\
z_{k+1}^{(2)}=-2\left(z_{k}^{(1)}\right)^{3}+2 z_{k}^{(1)}+0.3 y_{k} z_{k}^{(1)}
\end{array}\right.
$$

2) Step 2: The function $\left(z_{k}^{(1)}\right)^{3}$ is nonlinear and depend on the unmeasured state $z_{k}^{(1)}=x_{k}^{(1)}$. Then, we generate the new variable $z_{k}^{(3)}=\left(z_{k}^{(1)}\right)^{3}$ which leads to

$$
z_{k+1}^{(3)}=\left(z_{k+1}^{(1)}\right)^{3}=\left(z_{k}^{(2)}\right)^{3}
$$

The obtained system is then given in the form

$$
\left\{\begin{array}{l}
z_{k+1}^{(1)}=z_{k}^{(2)} \\
z_{k+1}^{(2)}=-2 z_{k}^{(3)}+2 z_{k}^{(1)}+0.3 z_{k}^{(1)} z_{k}^{(2)} \\
z_{k+1}^{(3)}=\left(z_{k}^{(2)}\right)^{3}
\end{array}\right.
$$

An in quasi-LPV form as follows

$$
\begin{aligned}
z_{k+1} & =\left[\begin{array}{ccc}
0 & 1 & 0 \\
2+0.3 y_{k} & 0 & -2 \\
0 & \left(y_{k}\right)^{2} & 0
\end{array}\right] z_{k} \\
y_{k} & =\left[\begin{array}{lll}
0 & 1 & 0
\end{array}\right] z_{k}
\end{aligned}
$$

By considering the premise variables

$$
\left\{\begin{array}{l}
\xi_{k}^{(1)}=y_{k} \\
\xi_{k}^{(2)}=\left(y_{k}\right)^{2}
\end{array}\right.
$$

and the fact that $0.4 \leq y_{k} \leq 1$, one obtains the TS model with measurable premise variables in the form (5). Then, by using the observer (6) one obtains the state estimation error dynamics (7). In this example the weighting functions depend only on the output $y_{k}$. Finally, by using the Lyapunov function $V\left(e_{k}\right)=e_{k}^{T} P e_{k}$ where $P=P^{T}>0$, simple LMI conditions are obtained as follows: If there exist a symmetric positive definite matrix $P$ and gain matrices $K_{i}, i=1, \ldots, 4$ such that the LMIs

$$
\begin{gathered}
{\left[\begin{array}{cc}
-P & \mathscr{A}_{i}^{T} P-\mathscr{C}^{T} K_{i}^{T} \\
P A_{i}-K_{i} \mathscr{C} & -P
\end{array}\right]<0} \\
i=1, \ldots, 4
\end{gathered}
$$

The gains of the observer are obtained by $\mathscr{L}_{i}=P^{-1} K_{i}, i=$ $1, \ldots, 4$. After solving these LMIs, the following gains are obtained

$$
\mathscr{L}_{1}=\mathscr{L}_{3}=\left[\begin{array}{l}
1 \\
0 \\
1
\end{array}\right], \mathscr{L}_{2}=\mathscr{L}_{4}=\left[\begin{array}{c}
1 \\
0 \\
0.16
\end{array}\right]
$$

The figure 1 illustrates the chaotic behavior of the Duffing map while the figure 2 shows the real states and the corresponding estimated states. Notice that estimating $z_{k}^{(1)}$ and $z_{k}^{(1)}$ corresponds to estimate $x_{k}^{(1)}$ and $x_{k}^{(1)}$ which means that no inverse transformation is needed.

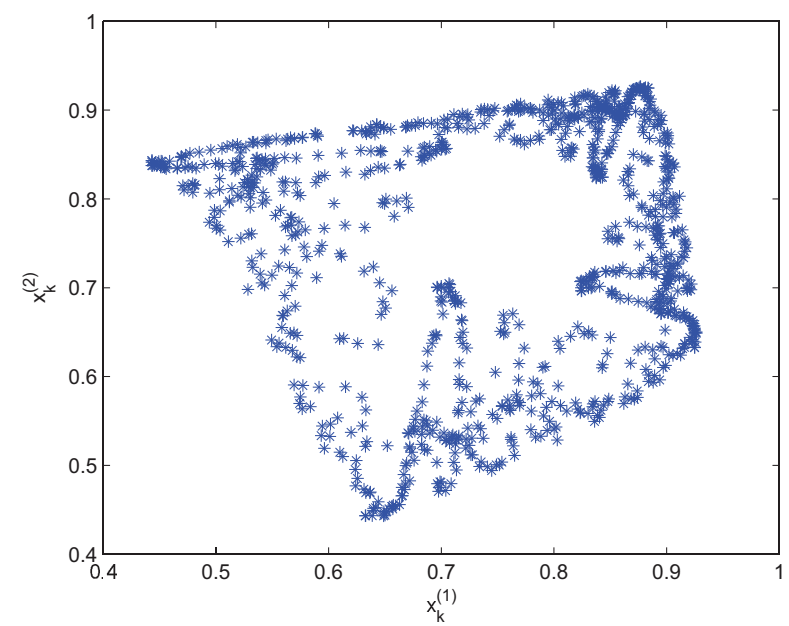

Fig. 1. Chaotic behavior of the Duffing map

Remark 1: An important remark is that the initial conditions can be chosen arbitrarily in this case because they do not affect the convex sum property. As can be seen in the case of unmeasurable premise variable TS systems, the unmeasured states are replaced by the estimated ones. Therefore, the initial conditions of the observer should be chosen in the compact set used to obtain the TS system. Notice that even if the initial conditions are in the compact 

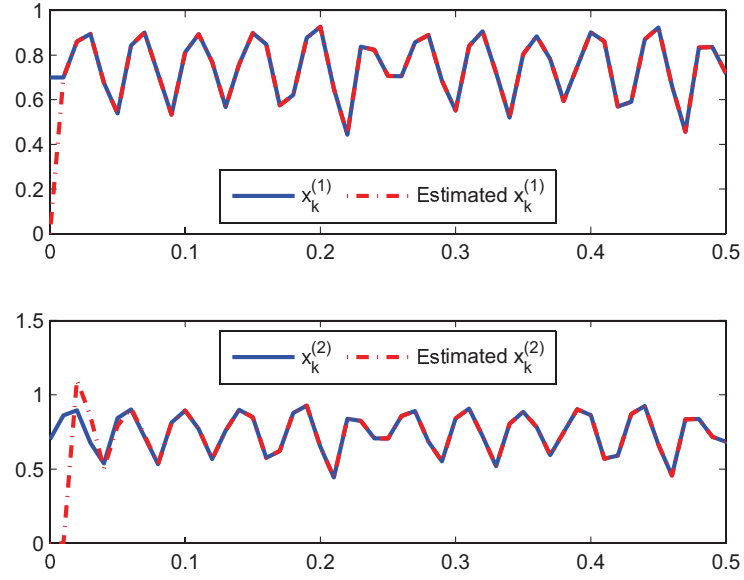

Fig. 2. State estimation

set, it may happen that the convex sum property will be lost in the transient phase and then the stability is no longer satisfied. By using the proposed approach, even if the initial conditions are chosen outside of the compact set (see the example), the convex sum property is always satisfied because the weighting functions depend only on the measured variable $y_{k}$ which varies in the domain $[0.4,1]$.

\section{B. Extension to PI observer design}

In the previous section, only state reconstruction objective is considered. The extension to the case of systems affected by constant or piecewise constant unknown inputs can be performed with the same algorithm. Indeed, since the unknown input is constant, its dynamic is described as $d_{k+1}=d_{k}$ which can be considered as an additional state in the original state vector. From this assumption, the same algorithm can be used to transform the system in the form (4) and then by using the sector nonlinear transformation for the obtained system, the TS form can be derived which has measurable premise variables.

Example 2: Let us consider the same chaotic system as in example 1 affected, in nonlinear manner, by the unknown input $d_{k}$ as follows

$$
\left\{\begin{array}{l}
x_{k+1}^{(1)}=x_{k}^{(2)} \\
x_{k+1}^{(2)}=-2\left(x_{k}^{(1)}\right)^{3}+2 x_{k}^{(1)}+0.3 x_{k}^{(1)} x_{k}^{(2)}+x_{k}^{(1)} d_{k} \\
y_{k}=x_{k}^{(2)}
\end{array}\right.
$$

It can be seen that the unknown input is multiplied by the unmeasured state $x_{k}^{(1)}$. By using the proposed algorithm, the following state transformation is obtained

$$
\left[\begin{array}{c}
z_{k}^{(1)} \\
z_{k}^{(2)} \\
z_{k}^{(3)} \\
z_{k}^{(4)}
\end{array}\right]=\left[\begin{array}{c}
x_{k}^{(1)} \\
x_{k}^{(2)} \\
\left(x_{k}^{(1)}\right)^{3} \\
x_{k}^{(1)} d_{k}
\end{array}\right]
$$

which leads to the rigorously equivalent system

$$
\left\{\begin{array}{l}
z_{k+1}^{(1)}=z_{k}^{(2)} \\
z_{k+1}^{(2)}=-2 z_{k}^{(3)}+2 z_{k}^{(1)}+0.3 z_{k}^{(1)} z_{k}^{(2)}+z_{k}^{(4)} \\
z_{k+1}^{(3)}=\left(z_{k}^{(2)}\right)^{3} \\
z_{k+1}^{(4)}=z_{k}^{(2)} d_{k}
\end{array}\right.
$$

which takes the quasi-LPV form

$$
\begin{aligned}
z_{k+1} & =\left[\begin{array}{cccc}
0 & 1 & 0 & 0 \\
2+0.3 y_{k} & 0 & -2 & 1 \\
0 & \left(y_{k}\right)^{2} & 0 & 0 \\
0 & 0 & 0 & 0
\end{array}\right] z_{k}+\left[\begin{array}{c}
0 \\
0 \\
0 \\
y_{k}
\end{array}\right] d_{k} \\
y_{k} & =\left[\begin{array}{llll}
0 & 1 & 0 & 0
\end{array}\right] z_{k}
\end{aligned}
$$

The matrices of the obtained model depend only on the measured output and by considering the premise variables $\xi_{k}^{(1)}=y_{k}$ and $\xi_{k}^{(2)}=\left(y_{k}\right)^{2}$, a TS model with measurable premise variables is obtained in the form

$$
\left\{\begin{array}{l}
z_{k+1}=\sum_{i=1}^{4} h_{i}\left(y_{k}\right)\left(\mathscr{A}_{i} z_{k}+\mathscr{B}_{i} d_{k}\right) \\
y_{k}=\mathscr{C} z_{k}
\end{array}\right.
$$

In order to estimate the state vector $z_{k}$ and the unknown input $d_{k}$, the following PI observer can be used

$$
\left\{\begin{array}{l}
\hat{z}_{k+1}=\sum_{i=1}^{4} h_{i}\left(y_{k}\right)\left(\mathscr{A}_{i} \hat{z}_{k}+\mathscr{B}_{i} \hat{d}_{k}+\mathscr{L}_{i}\left(y_{k}-\hat{y}_{k}\right)\right) \\
\hat{d}_{k+1}=\hat{d}_{k}+\sum_{i=1}^{4} h_{i}\left(y_{k}\right) \mathscr{H}_{i}\left(y_{k}-\hat{y}_{k}\right) \\
\hat{y}_{k}=\mathscr{C} \hat{z}_{k}
\end{array}\right.
$$

By considering the state and unknown input estimation errors $e_{k}=z_{k}-\hat{z}_{k}$ and $s_{k}=d_{k}-\hat{d}_{k}$, one obtains

$$
\left[\begin{array}{l}
e_{k+1} \\
s_{k+1}
\end{array}\right]=\sum_{i=1}^{4} h_{i}\left(y_{k}\right)\left[\begin{array}{cc}
\mathscr{A}_{i}-\mathscr{L}_{i} \mathscr{C} & \mathscr{B}_{i} \\
\mathscr{H}_{i} \mathscr{C} & I
\end{array}\right]\left[\begin{array}{c}
e_{k+1} \\
s_{k+1}
\end{array}\right]
$$

Then the stability of the system generating the state and unknown input errors can be studied by the classical Lyapunov tools developed for TS systems with measurable premise variables. In figures 3 and 4 , both state and unknown input reconstruction are illustrated. It can be seen that the states and the unknown input are estimated asymptotically.

\section{Discussions}

The proposed approach is an answer to the question asked in the introduction of this paper. From the examples, it can be seen that it is possible to avoid the unmeasurable premise variables in the TS model by extending the state vector of the original system before using the nonlinear sector transformation. Then, the classical techniques of observer design for TS systems with measurable premise variables can be used. The proposed approach is adequate for nonlinear systems where applying, directly, the sector nonlinear transformation provides inevitably a TS system with unmeasurable premise variables. In the following, some points are discussed: 

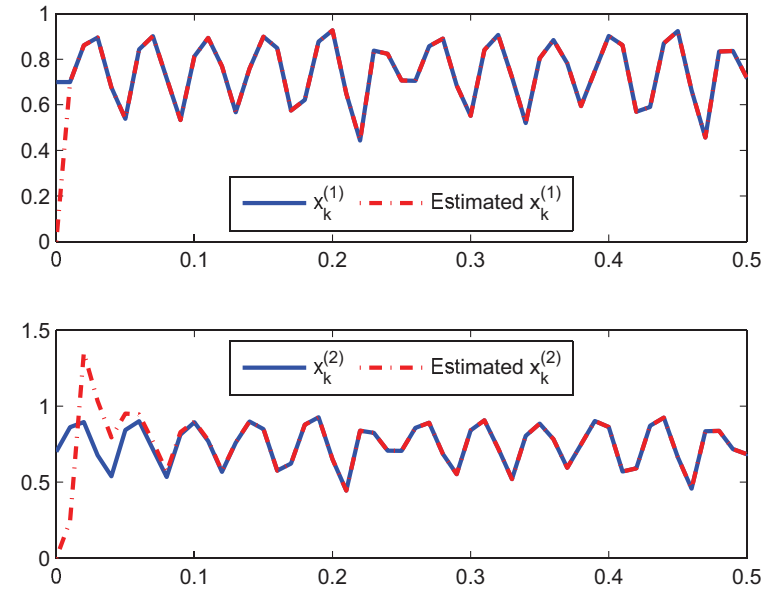

Fig. 3. State reconstruction in the presence of unknown input

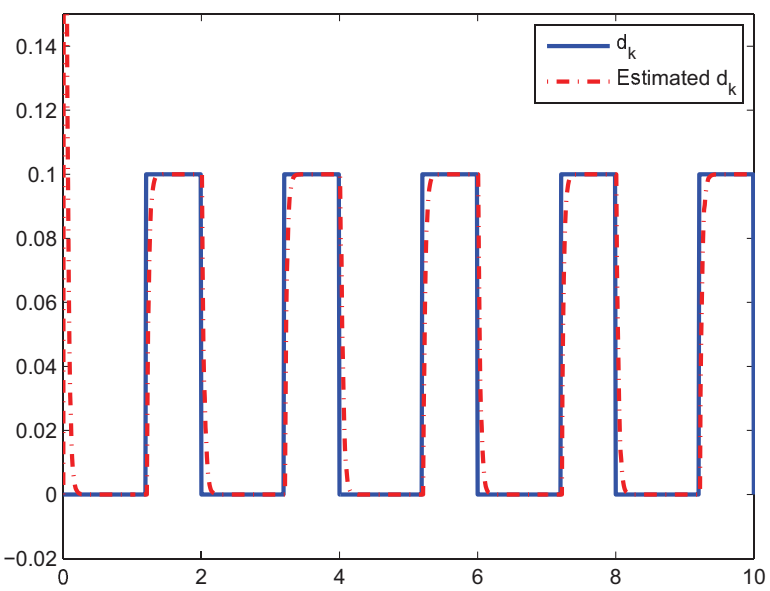

Fig. 4. Unknown input reconstruction

- Observer design for TS systems with unmeasurable premise variables is done by replacing the unmeasured states involved in the premise variables by the estimated version. However, a particular attention should be taken into account on the initial values of the observer which must be in the compact set where the TS model is valid in order to ensure that the convex sum property of the weighting functions is satisfied. Unfortunately, in some cases, even if the initial conditions of the observer belong to the compact set, the convex sum property may be lost in transient phase which may cause the non negativity of the Lyapunov function variations and then, the stability is no longer guaranteed. The proposed approach solves this problem since the premise variables depend on the measured variables which belong to a compact set and do not change for different initial conditions (see the examples 1 and 2).

- As a comparison to existing immersion techniques in nonlinear systems, these lasts aim to immerse the orig- inal system in a new state space where the obtained systems present a particular structure. In addition, the classical immersion techniques should be invertible in order to express the states of the original system in respect to the states of the immersed one. The presented algorithm aims only to express the system as a LPV system with parameters depending on measured variables and without any other particular structure for the matrices of the LPV. Furthermore, the proposed algorithm leads to a new state vector containing the states of the original system, consequently, there is no need to inversion of the transformation to recover the original states.

- As for nonlinear sector transformation technique, the dynamic state extension, presented in this paper, is not unique. Then, the dynamic state extension should be judiciously chosen in such a way to preserve the observability property of the original system which is a disadvantage compared to existing nonlinear immersion techniques. Indeed, the immersion techniques transform the system into an adequate new system with particular structure ensuring the observability.

- Notice also that the proposed algorithm may present an infinite number of iterations. For example, let us consider the system

$$
\left\{\begin{array}{l}
x_{k+1}^{(1)}=x_{k}^{(2)} \\
x_{k+1}^{(2)}=\left(x_{k}^{(2)}\right)^{2} \\
y_{k}=x_{k}^{(1)}
\end{array}\right.
$$

By applying the proposed algorithm, the following new states are obtained

$$
\begin{aligned}
& z_{k}^{(3)}=\left(z_{k}^{(2)}\right)^{2} \\
& z_{k}^{(4)}=\left(z_{k}^{(2)}\right)^{4} \\
& z_{k}^{(5)}=\left(z_{k}^{(2)}\right)^{8}
\end{aligned}
$$

which illustrates that the algorithm presents infinite number of iterations, then, there is no solution for this system. However, a solution can be obtained by using our previous work in [7] by using the algebraic technique.

From these discussions, the proposed algorithm provides a first solution to the problem of transformation of TS systems with unmeasurable premise variable into an equivalent TS system with measurable premise variables of dimension greater than or equal to the dimension of the original TS system. As illustrated by the examples, it can provide interesting results. The discussed points above presents research directions in order to obtain a systematic algorithm to obtain equivalent TS system with measured premise variables and preserves some properties of the original system such as observability and detectability. In addition, it is interesting to study the conditions under which such a transformation exists which avoids the infinite number of iterations. 


\section{CONCLUSIONS}

In this paper, a new technique is proposed in order to avoid the unmeasurable premise variables in the TS systems obtained from a nonlinear model of the system. Classically, the nonlinear models are transformed directly by using the sector nonlinear transformation which often lead to TS systems with premise variables depending on unmeasured states. It is known that dealing with the problem of observer design in such a situation is more complex than using TS systems with measurable premise variables. It is then natural to ask the question whether from the original nonlinear model it is possible to obtain an equivalent nonlinear model for which the nonlinear sector transformation provides a TS system with premise variables depending only on measured variables (input and output). This paper provides a solution by using the dynamic state extension known as immersion techniques in nonlinear framework. The difference between the known immersion techniques and the proposed one is that the state of the original system is contained in the new state vector which avoids the inversion of the immersion. An extension of the proposed approach for systems affected by unknown inputs is provided. For future work, a deep analysis of this approach will be performed in order to extend it to more general systems. In addition, since the transformation is not unique, the study will focus on the invariance property in order to obtain equivalent nonlinear model that preserves some properties of the original system such as observability and detectability.

\section{REFERENCES}

[1] P. Bergsten, R. Palm, and D. Driankov. Fuzzy observers. In IEEE International Fuzzy Systems Conference, Melbourne, Australia, 2001.

[2] G. Besancon. Nonlinear observers and applications. Lecture Notes In Control And Information Sciences (LNCIS), Springer, 2007.

[3] J. Daafouz, P. Riedinger, and C. Iung. Stability analysis and control synthesis for switched systems: a switched Lyapunov function approach. IEEE Transactions on Automatic Control, 47(11):1883-1887, Nov 2002.

[4] F. Gang. Stability analysis of discrete-time fuzzy dynamic systems based on piecewise lyapunov functions. IEEE Transactions on Fuzzy Systems, 12(1):22-28, Feb 2004

[5] K. Gasso, G. Mourot, and J. Ragot. Structure Identification in Multiple Model Representation: Elimination and Merging of Local Models. In 40th IEEE Conference on Decision and Control, pages 2992-2997, Orlando, United States, December 2001.

[6] D. Ichalal, H. Arioui, and S. Mammar. Observer design for twowheeled vehicle: A Takagi-Sugeno approach with unmeasurable premise variables. In 19th Mediterranean Conference on Control Automation, pages 934-939, Corfu, Greece, June 20-23 2011.

[7] D. Ichalal, S. Mammar, M. Dabladji, and J. Ragot. Observer design for a class of discrete-time quasi-LPV systems with unknown parameters: algebraic approach. In 14th European Control Conference, ECC'15, Linz, Austria, July 2015.

[8] D. Ichalal, B. Marx, D. Maquin, and J. Ragot. Observer design and fault tolerant control of Takagi-Sugeno nonlinear systems with unmeasurable premise variables. In Gerasimos Rigatos, editor, Fault Diagnosis in Robotic and Industrial Systems, pages 1-21. iConceptPress, 2012. Chapter 5, 21pages.

[9] D. Ichalal, B. Marx, J. Ragot, and D. Maquin. State and unknown input estimation for nonlinear systems described by Takagi-Sugeno models with unmeasurable premise variables. In 17th Mediterranean Conference on Control and Automation, Thessaloniki, Greece, June 24-26 2009.

[10] D. Ichalal, B. Marx, J. Ragot, and D. Maquin. State estimation of Takagi-Sugeno systems with unmeasurable premise variables. IET Control Theory \& Applications, 4(5):897-908, 2010.
[11] D. Ichalal, B. Marx, J. Ragot, and D. Maquin. Advances in observer design for Takagi-Sugeno systems with unmeasurable premise variables. In 20th Mediterranean Conference on Control Automation, pages $848-853$, july 2012 .

[12] Z. Lendek, R. Babuska, and B. De Schutter. Stability of cascaded fuzzy systems and observers. IEEE Transactions on Fuzzy Systems, 17(3):641-653, June 2009.

[13] R. Murray-Smith and T.A. Johansen. Multiple Model Approaches to Modelling and Control. Taylor and Francis, London, 1997.

[14] T. Takagi and M. Sugeno. Fuzzy identification of systems and its applications to modeling and control. IEEE Transactions on Systems, Man, and Cybernetics, 15:116-132, 1985

[15] K. Tanaka and H.O. Wang. Fuzzy Control Systems Design and Analysis: A Linear Matrix Inequality Approach. John Wiley and Sons, 2001.

[16] F.E. Thau. Observing the state of non-linear dynamic systems. International Journal of Control, 18:471-479, 1973.

[17] Z. Yacine, D. Ichalal, N.A. Oufroukh, S. Mammar, and S. Djennoune. Nonlinear vehicle lateral dynamics estimation with unmeasurable premise variable Takagi-Sugeno approach. In 20th Mediterranean Conference on Control Automation, pages 1117 -1122, July 2012. 